\title{
Remarks on the Erroneous Dispersion Surfaces from a Pair of a Hyperbolic Branch and an Elliptical Arc of the Intersected Two Laue Spheres Based on the Usual Crude Approximation
}

\author{
Tetsuo Nakajima \\ Saitama Institute of Technology, Advanced Science Research Laboratory, Saitama, Japan \\ E-mail:tetsuo_nakajima@y7.dion.ne.jp \\ Received February 5, 2011; revised March 7, 2011; accepted March 8, 2011
}

\begin{abstract}
In almost all previous works, the hyperbolic dispersion surfaces of the central proper quadrics have been crudely derived from reduction of the degree from the bi-quadratic equation by use of some roughly indefinable approximate relations. Moreover, neglecting the high symmetry of the hyperbola, both the branches have been approximated on the asymmetric surfaces composed of a pair of a branch of the hyperbola and a vertex of the ellipse without the presentation of reasonable evidence. Based upon the same dispersion surfaces equation, a new original gapless dispersion surfaces could be rigorously introduced without crude omission of even a term in the bi-quadratic equation based upon usual analogy with the extended band theory of solid as the close approximation to the truth.
\end{abstract}

Keywords: Dynamical Theory of X-Ray Diffraction, Gapless Dispersion Surface, Gappy Dispersion Surface

\section{Introduction}

First of all, it could be necessarily considered that the firm establishment of $E$ (energy) vs. $k$ (wave number) curves as the dispersion relation of the electron in solids, which have been used as the usually popular gappy dispersion surfaces by solid line in Figure 1 [1] in almost all works of the dynamical theory of X-ray diffraction (DTXD) [2-6], were carefully introduced from the solutions of the secular equation based upon the experimental and theoretical examinations in the low-energy electron-diffraction by R. M. Stern et al. [1] and have prevented to foster greater understanding of DTXD. In the band theory of the solid state physics, the energy gap at the Brillouin zone boundary between the hyperbola and ellipse in Figure 1 could be introduced as a perturbative effect of the Fourier component of the periodic potential in the crystal [7], which is the off-diagonal term in the secular equation shown in the Section 5. It plays important role that the band structure and its electronic structure occupied by electrons depending on their concentration could give an insulator, a metal or a semiconductor and a semi-metal [7]. The energy band structure with the forbidden band as the Bragg gap due to the potential barrier from the band theory in Figure 1 [1] could be valid for only the valence and conduction electrons and incident electrons in the electron diffraction $[1,7,10]$. On the contrary, the off-diagonal terms of $K^{2} \mathrm{C} \chi_{\bar{g}}$ and $K^{2} \mathrm{C} \chi_{g}$ in Equation (2) in the Section 3 are composed of the wave number, the polarization factor and the Fourier component of polarizability as a response function of X-rays, which could be rewritten by $\chi_{g}=R \lambda^{2} F_{g} / \pi V$ [3]. Here $R$ is the classical radius of electron, $\lambda$ the wavelength, $F_{g}$ the Fourier component of the structure factor and $V$ the unit cell volume. All of the factors are transparent to $\mathrm{X}$-rays contrary to the potential for electron, and therefore cannot construct forbidden band as the Bragg gap, as in the Section $2[8,9]$.

Secondly, the hyperbolic gappy dispersion surfaces in DXTD have been arranged near an intersectional point at the Lorentz point $L_{D}$ between the hyperbola and ellipse in Figure 1. The hyperbola is perfectly point symmetry about the center of the hyperbola. However, the gap in Figure 1 is composed of a branch of the hyperbola and an arc of the ellipse. According to each definition of the 


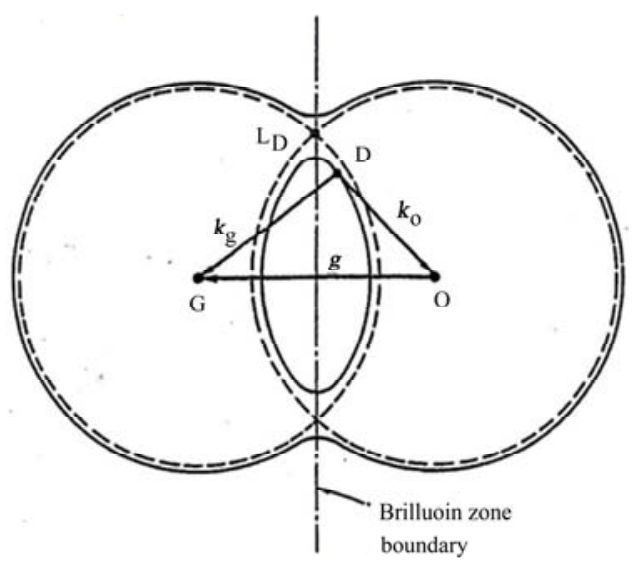

Figure 1. The familiar dispersion surfaces. The formation of the Bragg gap has reasonably derived only in the electron-diffraction by Stern [1]. Superposition of the hyperbola over the gap between a branch of hyperbola and a vertex of ellipse in DTXD is a misapplication by a lighthearted interpretation.

central proper quadrics, the ellipse and hyperbola can be formed from the loci that the sum and difference of the two distances from the definite two points are constant, respectively, as is written like $(x / a)^{2} \pm(y / b)^{2}=1$. Therefore, the hyperbola has two asymptotes that cannot exist in the ellipse. Let it be ever so infinitesimal line elements in each, ellipse and hyperbola are invariably ellipse and hyperbola, respectively. Generally, approximation can round off magnitude of the quantity but cannot change the plus and minus signs in algebra and the symmetry in geometry. Therefore, the ellipse could not transform into the hyperbola by approximation. By nature, that's something that cannot be, as mentioned in the sections 3 and 4.

As a preliminary work, surpassing the above two erroneous points the rightly reasonable dispersion surfaces [8,9] are carefully described based upon high analogy [3] with the extended band theory in solid state physics as the closest approximation to the truth [10].

\section{The First New Gapless Dispersion Surfaces in DTXD Based upon the Band Theory of the Solid}

Mainly following Kato's scheme [2] based upon a manner of the Laue method [3], we carefully examined derivation of the gapless dispersion surfaces from the biquadratic equation of the two wave approximation. The vector propagation equation of an electro-magnetic wave by the electric displacement $\boldsymbol{d}$ in a medium with a periodic polarizability $\chi(\mathbf{r})$ has been represented by

$$
\Delta d+K^{2} \boldsymbol{d}+\operatorname{rot} \cdot \operatorname{rot} \chi \boldsymbol{d}=0 .
$$

Based upon this equation, the two wave propagation equations from the Bloch waves of $\boldsymbol{d}_{\mathbf{0}}$ and $\boldsymbol{d}_{g}$ defined by

$$
\boldsymbol{d}(\boldsymbol{r})=d_{o} \exp \left(-i \boldsymbol{k}_{o} \cdot \boldsymbol{r}\right)+\boldsymbol{d}_{g} \exp \left(-i \boldsymbol{k}_{g} \cdot \boldsymbol{r}\right)
$$

could be derived to be

$$
\left(k^{2}-\boldsymbol{k}_{o}^{2}\right) d_{o}-K^{2} C \chi_{\bar{g}} d_{g}=0
$$

and

$$
K^{2} C \chi_{g} d_{\mathrm{o}}-\left(k^{2}-\boldsymbol{k}_{g}^{2}\right) d_{g}=0
$$

in which $k$ is the wave number in the crystal, $K$ the wave number in vacuum defined by $\left(k=K\left(1+\frac{\chi_{0}}{2}\right)=n K\right)$, where $n$ the refraction index, $C$ the polarization factor and $\chi_{\mathrm{o}, \pm g}$ Fourier components of the polarizability, in which $\chi_{g} \chi_{\bar{g}}=\left|\chi_{g}\right|^{2}$ from $\chi_{\bar{g}}=\chi_{g}^{*}$ by neglecting the absorption. Here, in order to solve the two wave propagation Equations (1a) and (1b), which could be represented by the simultaneous linear equations with two unknowns, the necessary and sufficient condition, which is satisfied for existence of solutions [6] could be represented by

$$
\begin{gathered}
\left|\boldsymbol{S}_{\mathrm{ij}}\right|=\left|\begin{array}{cc}
\mathrm{k}^{2}-\boldsymbol{k}_{\mathrm{o}}^{2} & K^{2} \mathrm{C} \chi_{\overline{\mathrm{g}}} \\
K^{2} \mathrm{C} \chi_{g} & \mathrm{k}^{2}-\boldsymbol{k}_{g}^{2}
\end{array}\right|=\mathrm{k}^{4}-\left(\boldsymbol{k}_{\mathrm{o}}^{2}+\boldsymbol{k}_{g}^{2}\right) \mathrm{k}^{2} \\
+\boldsymbol{k}_{\mathrm{o}}^{2} \cdot \boldsymbol{k}_{g}^{2}-K^{4} \mathrm{C}^{2} \chi_{g}^{2}=0,
\end{gathered}
$$

which was designated as the bi-quadratic dispersion surface equation called by Pinsker [4], not the secular equation. Here, heads of $k_{\mathrm{o}}$ and $k_{g}\left(\equiv k_{\mathrm{o}}+g\right.$ where $g$ is the reciprocal lattice vector) in Figure 2 lie at the point $O$ and $G$ and their initial common point $S_{1}$, which chanced to be there satisfies a loci in Equation (2) in the

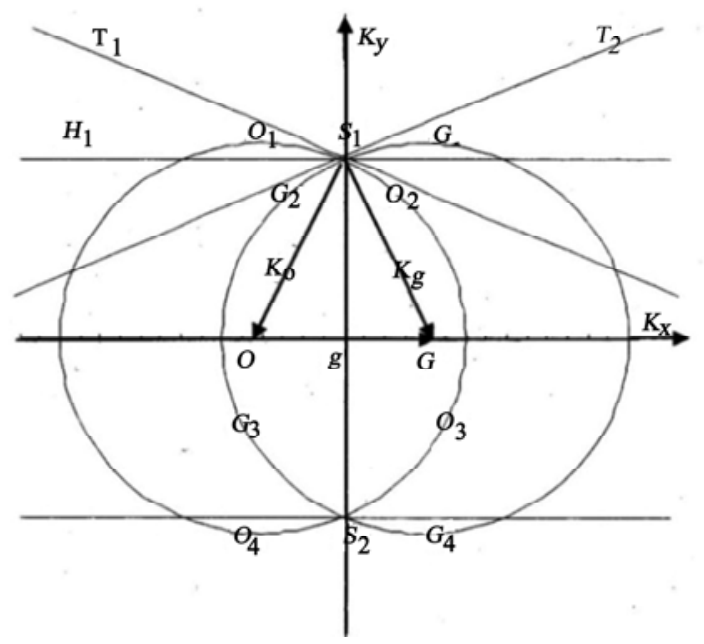

Figure 2. Diffraction in reciprocal space based upon the two wave approximation. The points $S_{1}$ and $S_{2}$ are intersectional points, through which a horizontal lines of $H_{1}$ and $H_{2}$ are reference lies. The vector of $\overrightarrow{O G}=g$ is the reciprocal lattice vector, $\overrightarrow{S_{1} O}=k_{o}$ the incident wave vector and $\overrightarrow{S_{1} G}=\boldsymbol{k}_{g}$ the reflected wave vector. 
reciprocal space.

The diagonal terms of $S_{11}$ and $S_{22}$ in Equation (2) represent the two same radius circles intersected at two points $S_{1}$ and $S_{2}$ in Figure 2. The roots of $X\left(=k^{2} \geq 0\right.$ : positive definite) in Equation (2) can be given by

$$
X=\frac{1}{2}\left\{\left(\boldsymbol{k}_{\mathrm{o}}^{2}+\boldsymbol{k}_{g}^{2}\right) \pm \sqrt{\left(\boldsymbol{k}_{\mathrm{o}}^{2}-\boldsymbol{k}_{g}^{2}\right)^{2}+4 K^{4} C^{2} \chi_{g}^{2}}\right\} .
$$

Accurately, Equation (2) could not be a form of the secular equation but has been frequently impressed as the secular equation in some references $[2,3,6]$. Just to be sure, the intrinsic secular equation of equation (2) could be represented from the usual definition as follows:

$$
\begin{aligned}
& \operatorname{Det}\left\{\left(\boldsymbol{S}_{\mathrm{ij}}\right)-\boldsymbol{E}\left(\delta_{i j}\right)\right\}=\left|\begin{array}{cc}
\boldsymbol{k}_{\mathbf{o}}^{2}-k^{2}-E & K^{2} C \chi_{\bar{g}} \\
K^{2} C \chi_{g} & \boldsymbol{k}_{g}^{2}-k^{2}-E
\end{array}\right| \\
& =E^{2}-\left(\boldsymbol{k}_{\mathrm{O}}^{2}+\boldsymbol{k}_{g}^{2}-2 k^{2}\right) E+\boldsymbol{k}_{\mathrm{O}}^{2} \cdot \boldsymbol{k}_{g}^{2}- \\
& \left(\boldsymbol{k}_{\mathrm{O}}^{2}+\boldsymbol{k}_{g}^{2}\right) k^{2}+k^{4}-\left(K^{2} C \chi_{g}\right)^{2}=0,
\end{aligned}
$$

in which $E$ is the eigen-value, $\delta_{i j}=\left\{\begin{array}{ll}1 & \text { for } i=j \\ 0 & \text { for } i \neq j\end{array}\right.$ the Kronecker's delta, which represents the unit matrix. The eigen-values of Equation (4) could be obtained to be

$$
E=-k^{2}+\frac{1}{2}\left\{\left(\boldsymbol{k}_{\mathrm{o}}^{2}+\boldsymbol{k}_{g}^{2}\right) \pm \sqrt{\left(\boldsymbol{k}_{\mathrm{o}}^{2}-\boldsymbol{k}_{g}^{2}\right)^{2}+4 K^{4} C^{2} \chi_{g}^{2}}\right\},
$$

where the first term of " $-k^{2}$ "in Equation (5) only translates the origin of $E$, therefore ordinarily it is omitted hereafter. It is the most important that the behavior of the whole multinomial commonly in the right hand side in Equations (3) and (5) should be carefully analyzed over all $k$-space especially in the vicinity of the Brillouin zone boundary. When $E=0$, Equations (2) and (3) are completely equivalent to Equations (4) and (5), respectively. Then, it is self-evident that the analytic results in both of Equations (3) and (5) without any abbreviation of even a term as done in the conventional crude approximation could remain wholly valid as the close approximation to the truth. It could be understood that the refracted beam $k$ in the roots in Equation (3) can be sufficiently characterized by AM and FM due to the three kinds of photons of $\boldsymbol{k}_{\mathrm{o}}, \boldsymbol{k}_{g}$ and $K$, which could be polarized by the periodic electron distribution in $\chi_{g}$ in conformity with the dual polarized photons by $C$.

Approximately assuming that $K^{4} C^{2} \chi_{g}^{2} \cong 0$, two intersected circles with the same radii in Equation (3) are shownin Figure 2. If the magnitudes of $k_{\mathrm{o}}$ and $k_{g}$ are close to each other, then the term of $4 K^{4} C^{2} \chi_{g}^{2}$ cannot be neglected. Thus, the amplitude of neither plane waves is negligible. When $\left|k_{\mathrm{o}}\right|=\left|k_{g}\right|$, Equation (3) becomes $X=k_{\mathrm{o}}^{2} \pm K^{2} C \chi_{g}$ and hence the ratio of $d_{o}$ to $d_{g}$, determined from Equation (2) is $K^{2} C \chi_{\overline{\mathrm{g}}}: \pm\left|K^{2} C \chi_{\bar{g}}\right|$ Hence, $\left|d_{\mathrm{o}}\right|:\left|d_{g}\right|=1: 1$. Assuming that $4\left(K^{2} C \chi_{g}\right)^{2}$ is large compared with the first term under the radical sign in Equation (3) in case of $\left|\boldsymbol{k}_{\mathrm{o}}^{2}\right| \cong\left|\boldsymbol{k}_{g}^{2}\right|, X$ can be expanded to be,

$$
X=\frac{1}{2}\left(\boldsymbol{k}_{\mathrm{o}}^{2}+\boldsymbol{k}_{g}^{2}\right) \pm K^{2} C \chi_{g} \pm\left\{\left(\boldsymbol{k}_{\mathrm{o}}^{2}-\boldsymbol{k}_{g}^{2}\right)^{2} / 8 K^{2} C \chi_{g}\right\} \pm \cdots
$$

If we translate the origin of $\boldsymbol{k}$ by $-\boldsymbol{g} / 2$ and consider the vector $\boldsymbol{k}+\boldsymbol{g} / 2$ and if we denote by $\boldsymbol{x}$ the component of $k$ parallel to $-g$ and by $z$ the normal component to $-g$, then by using the following relations, after more elaborate vector analysis than it looks like,

$$
\begin{gathered}
\boldsymbol{k}_{g}^{2}=\boldsymbol{k}_{\mathrm{o}}^{2}+2 k_{o} \cdot \boldsymbol{g}+\boldsymbol{g}^{2}=\boldsymbol{k}_{\mathrm{o}}^{2}+ \\
2\left\{z+\left(\boldsymbol{x}-\frac{\boldsymbol{g}}{2}\right)\right\} \cdot \boldsymbol{g}+\boldsymbol{g}^{2}=\boldsymbol{k}_{\mathrm{o}}^{2}+2 \boldsymbol{x} \cdot \boldsymbol{g}
\end{gathered}
$$

and

$$
\boldsymbol{k}_{\mathrm{o}}^{2}=\boldsymbol{z}^{2}+\left(\boldsymbol{x}-\frac{\boldsymbol{g}}{2}\right)^{2}=\boldsymbol{z}^{2}+\boldsymbol{x}^{2}-\boldsymbol{x} \cdot|\boldsymbol{g}|+|\boldsymbol{g}|^{2} / 4,
$$

a reasonable roots of $X$ in Equation (3) from the 2nd and 3rd terms in Equation (6) can be rewritten as

$$
\begin{gathered}
X=\boldsymbol{z}^{2}+\boldsymbol{x}^{2}+\boldsymbol{g}^{2} / 4 \\
\pm\left[\left|K^{2} C \chi_{g}\right| \pm \boldsymbol{x}^{2} /\left(2\left|K^{2} C \chi_{g}\right| / \mathbf{g}^{2}\right)\right] \pm \cdots
\end{gathered}
$$

The $4^{\text {th }}$ term in the brackets in Equation (7) could be the most important one expanded in series near the Brilluoin zone boundary described in the above as one of main subjects. As a result, the $4^{\text {th }}$ term in Equation (7) could be indicated by

$$
\boldsymbol{y}^{2}=\left[K^{2} C \chi_{g} \pm\left(\boldsymbol{g}^{2} \boldsymbol{x}^{2} / 2 K^{2} C \chi_{g}\right)\right] .
$$

The expressions in Equation (8) show the precisely canonical forms of the hyperbola and ellipse as

$$
y^{2}=b^{2} \pm\left(b^{2} x^{2} / a^{2}\right)
$$

in which the ellipse with plus sign and hyperbola with minus one are shown in Figures 3(a) and 3(b), together with asymptotes of $\frac{y}{b} \pm \frac{x}{a}=0$ labeled $L_{1}$ and $L_{2}$ in hyperbola. The constants $a$ and $b$ in Equation (9) can be given by Equation (8) as

$$
a=\sqrt{2} K^{2} C \chi_{g} / g
$$



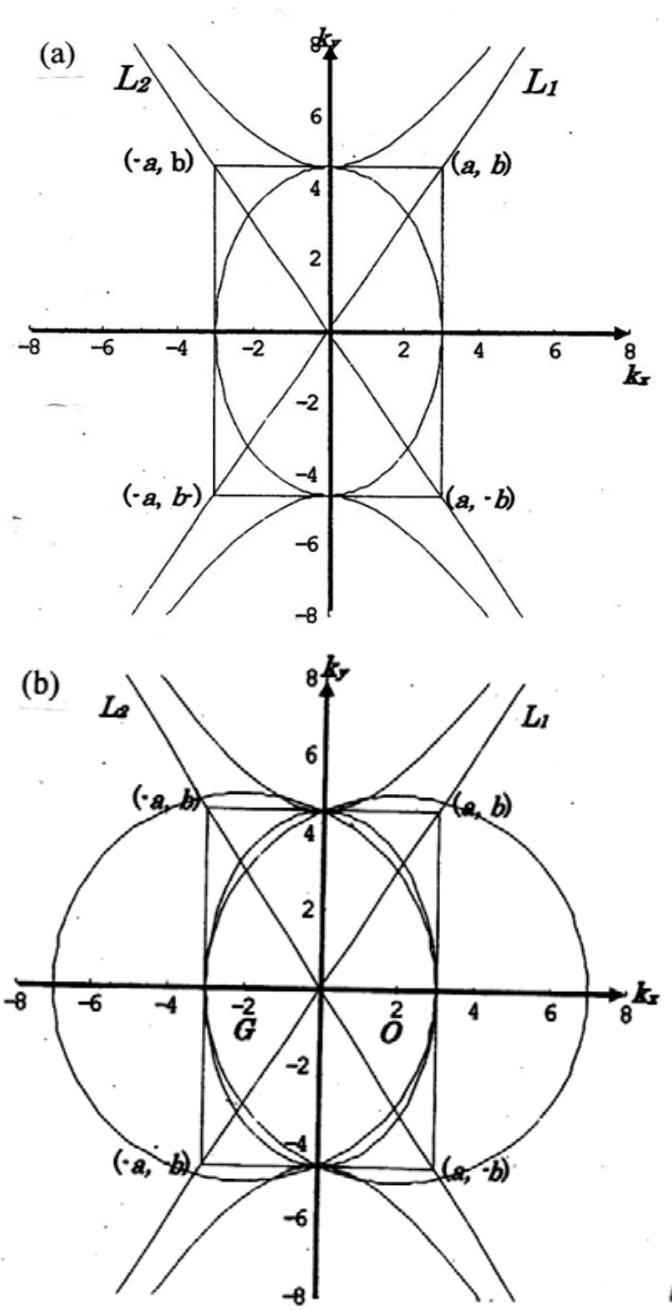

Figure 3(a). The dispersion surfaces composed of the ellipse and hyperbola in Equation (8) with the two asymptotes $L_{1}$ and $L_{2}$ defined by extended two diagonals of the rectangle with both sides of $2 a$ and $2 b$. 3(b).This shows superposition of Figure 3(a) upon Figure 1, which shows smooth variations of the dispersion surfaces in forward and backward $X$-ray near the Brillouin zone boundary.

$$
b=K \sqrt{C \chi_{g}} .
$$

Therefore, $2 a=2 \sqrt{2} K^{2} C \chi_{g} / g$ and $2 b=2 K \sqrt{C \chi_{g}}$ from Equations (10a) and (10b) are the minor and major axes of the ellipse, respectively and the latter of $2 b$ also stands for the transverse axis of the hyperbola. The gradients of the two asymptotes of hyperbola defined by the gradients of diagonals of the rectangle with both sides of $2 a$ and $2 b$ in Figures 3(a) and 3(b) could be expressed by $\pm(\mathrm{b} / \mathrm{a})= \pm \sqrt{2 / C \chi_{g}} \cdot \sin \theta_{B}$ from Equations (8) and (9) by the Bragg law, which will be discussed in the final section. Both of the hyperbola and ellipse could stand in a line without a gap as in Figures 3(a) and 3(b). Conse- quently, it could be apparently proved that the expected Bragg gap as shown in Figure 1 between hyperbola and ellipse could not absolutely exist in Equation (8) in DTXD and the gappy dispersion surfaces in Figure 1 can be rigorously set to the right gapless dispersion surfaces in Figure 3(a).

\section{Examination on the Crude Approximation to Derive the Previous Gappy Dispersion Surfaces}

According to the previous works [2-6], the dispersion surface Equation (2) can be factorized as

$$
\begin{gathered}
\left|\begin{array}{cc}
\boldsymbol{k}_{\mathbf{o}}^{2}-k^{2} & K^{2} C \chi_{\bar{g}} \\
K^{2} C \chi_{g} & \boldsymbol{k}_{g}^{2}-k^{2}
\end{array}\right|= \\
\left(\boldsymbol{k}_{\mathbf{o}}+k\right)\left(\boldsymbol{k}_{g}+k\right)\left(\boldsymbol{k}_{\mathbf{o}}-k\right)\left(\boldsymbol{k}_{g}-k\right)-K^{4} C^{2} \chi_{g}^{2}=0 .
\end{gathered}
$$

In almost previous references [2-6], by use of the numerical approximate relations of

$$
k_{\mathrm{O}}+k \approx 2 K \approx 2 k \text { and } k_{g}+k \approx 2 K=2 k,
$$

the central proper bi-quadratic Equation (11) could be further decomposed into two quadratics as follows:

$$
\begin{gathered}
\left(\boldsymbol{k}_{\mathbf{o}}+k\right)\left(\boldsymbol{k}_{g}+k\right)-4 K^{2}=0 \\
\left(\boldsymbol{k}_{\mathbf{o}}-k\right)\left(\boldsymbol{k}_{g}-k\right)-\left(K^{2} C^{2} \chi_{g}^{2} / 4\right)=0 .
\end{gathered}
$$

The factorization in Equation (11) by Equation (12) is over the limits of approach and beyond reasonable conception in Equation (4).

Originally, the bi-quadratic dispersion surface Equation (2) should be a product of the two central proper quadrics consisting of the hyperbola and ellipse including circle as easily understood from Figures 1 and 2. First, the central proper quadric of the hyperbola in Equation (13a), which could be constructed by replacing a very big product of $k_{\mathrm{O}}+k$ and $k_{g}+k$ with $4 K^{2}$ by use of Equation (12), has been cut down without any thought for the consequence. Secondly, although a product of both terms of $k_{\mathbf{o}}-k$ and $k_{g}-k$ has been approximated at zero from Equation (12), an order of magnitudes of the second term in Equation 13(b) can be estimated to be

$$
\begin{aligned}
& K^{4} C^{2} \chi_{g}^{2} \gg K^{2} C^{2} \chi_{g}^{2} / 4 \cong\left(C^{2} / 4\right) \cdot 10^{2(9 \mathrm{to} 10)} \cdot 10^{-5 \times 2} . \\
& \cong\left(C^{2} / 4\right) \cdot 10^{8 \mathrm{to} 10} \gg 1
\end{aligned} .
$$

This is tremendously large number. The above approximate expression cannot coincide with Equations (11) and (13b). Commonly used factors in Equations (13a) and (13b) from Equation (12) could be only understandable in approximation of numerical values but not in vector 
analysis of $\boldsymbol{k}_{\mathbf{0}}$ and $\boldsymbol{k}_{g}$ considering its direction and orientation in the vector space. These approximations are unmistakably self-contradictory and definitely break native goodness of the two central proper composite quadrics in Equation (2), which should be never allowed without rigorous verification. This is a violation without cause.

Reserving examination of the elimination of the decomposed factors in Equation (13a) in the crude approximation, it could be concluded that Equation (13b) intuitively has at least two solutions. From the condition that the product of two variables is a constant of $\left(K^{2} C^{2} \chi_{g}^{2} / 4\right)$ in Equation (13b), a simple solution represents a rectangular hyperbola. However, this is not practically reasonable, considering the variations of the Bragg angle. In another solution, it could be easily understood from a well-known attribute that the product of the two perpendiculars to the two asymptotes of the hyperbola from an arbitrary point on it is constant described by $\left\{a^{2} b^{2} /\left(a^{2}+b^{2}\right)\right\}$, which could be easily prove from the canonical form of Equation (13b) as

$$
\begin{gathered}
\left(\frac{k_{\mathrm{y}}}{K C \chi_{g} / 2 \cos \theta_{\mathrm{B}}}\right)^{2}-\left(\frac{k_{\mathrm{x}}}{K C \chi_{g} / 2 \sin \theta_{\mathrm{B}}}\right)^{2}=1, \\
\left(0<\theta_{\mathrm{B}}<\pi / 2\right) .
\end{gathered}
$$

The fitness of Equation (14) to the hyperbola at the centure point $L_{\mathrm{D}}$ in Figure 1, which is composed of a hyperbolic branch (named as "branch 1 " for $\boldsymbol{k}_{\mathbf{0}}-k$, $\left.\boldsymbol{k}_{g}-k>0\right)$ and an elliptic arc near a vertex on the major axis side (similarly "branch 2" for $k_{\mathrm{o}}-k, k_{g}-k<0$ ) has not yet been correctly examined from a geometrical viewpoint to decide whether to support Equation (13b) on the principle of being fair and just.

\section{Geometrical Examination on the Conventional Gappy Dispersion Surfaces In DTXD}

It is thoughtless beyond mathematical knowledge to assimilate both substantially different extremities from a different nature of symmetry between hyperbola and ellipse, by simple numerical approximation in Figure 1. In Figure 2, the oblique lines of $T_{1}$ and $T_{2}$ represent tangential lines of two circles at the intersection point $S_{1}$. It is very important to note that the intersected curves above and below $H_{1}$ show quietly an obvious asymmetry, which could be paraphrased as follows: the vertically opposite angles at the intersectional point $S_{1}$ of the two composite arcs $O_{1} S_{1} G_{1}$ and $O_{2} S_{1} G_{2}$ defined by intersectional angle included by both of the tangential lines $T_{1}$ and $T_{2}$ at $S_{1}$ are identical. The angle included by two tangential lines at the points $O_{1}$ and $G_{1}$ on both $\operatorname{arcs} S_{1} O_{1}$ and
$S_{1} G_{1}$ can increase with increasing distances to both points $O_{1}$ and $G_{1}$ from the point $S_{1}$ and become monotonically larger than the vertically opposite angles in the vicinity of $S_{1}$. But a variation of the corresponding angles included by two tangential lines at the points $O_{2}$ and $G_{2}$ on both $\operatorname{arcs} S_{1} O_{2}$ and $S_{1} G_{2}$ can become entirely vice versa. As easily imagined from Figure 2, both of the whole closed curves of oval $S_{1} G_{2} G_{3} S_{2} O_{3} O_{2} S_{1}$ and cocoon-shaped $S_{1} O_{1} O_{4} S_{2} G_{4} G_{1} S_{1}$ constricted in the middle could be redrawn as hyperbolic and elliptic dispersion surfaces with the Bragg gap as in Figure 1 for lowenergy electron-diffraction by Stern [1], not for X-ray diffraction. This is a rigorous proof of asymmetry of arcs $O_{1} S_{1} G_{1}$ and $O_{2} S_{1} G_{2}$, from which the symmetric branches of the hyperbola can never be constructed. Therefore, the gappy dispersion surfaces in Figure 1 cannot be applied to TDXD as the hyperbola. In the previous works, a pair of asymmetric arcs of $O_{1} S_{1} G_{1}$ and $O_{2} S_{1} G_{2}$ has been insensitively replaced by the hyperbola of Equation (13b). This is the second violation without cause. For a long term, the previous works [2-6] have been just an attempt to put together the indefinable hybrid dispersion surfaces composed of a pair of the hyperbolic branch and elliptic arc to lay basis for today's TDXD. However, by nature that is something that cannot be. Consequently, approximation can round off magnitude of the quantity but cannot substantially change the plus and minus sign and geometrical symmetry. Therefore, the ellipse could not transform into the hyperbola by reasonably scientific approximation, whose use is totally outrageous and should not be fundamentally permitted.

\section{Origin of the Energy Gap in the Band Theory of Solid and Brief Characterization of the Off-Diagonal Term in the Dispersion Surface Equation (2)}

There are very close resemblance in physical treatment between the energy gap in conduction band $[1,7,10]$ and anomalous transmission of X-rays [2-6] but the definite necessary results reveal that the off-diagonal terms of the Fourier component of potential in electrons create a forbidden energy gap $[1,7,10]$ and those in photons in Equation (2) closely relate with the different ratio between the absorption and transmission of photons [2-6].

According to Kittel [7], two different standing waves of electron in solids could be derived from the two traveling waves of $\exp (i \pi x / a)$ and $\exp (-i \pi x / a)$ as

$$
\psi(+)=\exp \left(\frac{i \pi x}{a}\right)+\exp \left(-\frac{i \pi x}{a}\right)=2 \cos \pi x / a
$$


and

$$
\begin{aligned}
& \psi(-)=\exp \left(\frac{i \pi x}{a}\right)-\exp \left(-\frac{\mathrm{i} \pi x}{a}\right) \\
& =2 i \sin (\pi x / a) .
\end{aligned}
$$

The two standing waves $\psi(+)$ and $\psi(-)$ pile up electrons at different regions, and therefore the two waves have different values of the potential energy. This is the origin of the energy gap. The probability density $\rho$ of a particle is equal to $\psi^{*} \psi=\left|\psi^{2}\right|$. For a pure traveling wave $\exp \left(i^{k} x\right), \rho$ is equal to $\exp \left(i^{k} x\right) \exp (-i k x)=1$ so that the charge density is constant in Figure 4(b).

From the standing waves $\psi(+)$ in Equation (15a), the probability density could be expressed by

$$
\rho(+)=|\psi(+)|^{2} \propto \cos ^{2}(\pi x / a) .
$$

The function piles up negative charge on the positive ions at the periodic lattice points of $x=n a(n=0,1,2$, $\cdots)$, where the potential energy is the lowest. For the standing wave $\psi(-)$ in Equation (15b) the probability density is given by

$$
\rho(-)=|\psi(-)|^{2} \propto \sin ^{2}(\pi x / a),
$$

which concentrate electrons away from the ion cores.

Consequently, the wave function $\psi(+)$ piles up electronic charge on the cores of the positive ions, thereby lowering the potential energy in comparison with the average potential energy seen by a traveling wave in Figure 4(b). The wave function $\psi(-)$ piles up charge in the region between the ions, thereby raising the potential energy in comparison with that seen by a traveling wave in Figure 4(b). When the expectation values of the potential energy could be calculated over these three

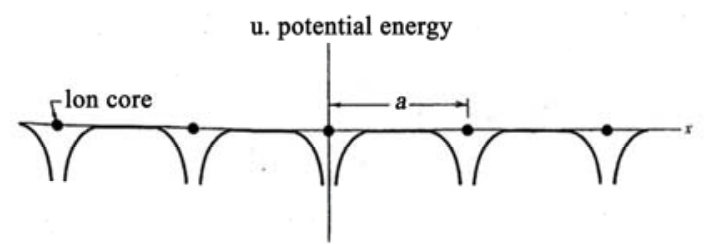

(a)

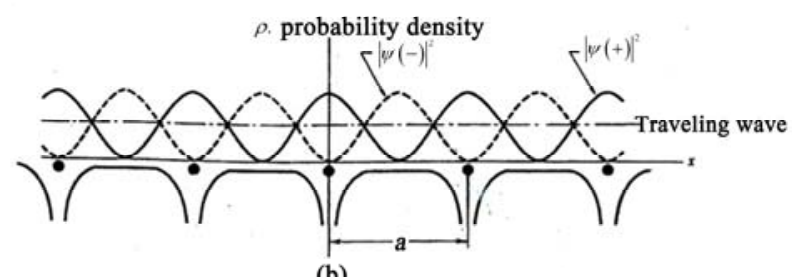

(b)

Figure 4(a) Variation of electrostatic potential energy of conduction electron in the field of ion cores of a linear lattice. 4(b) Distribution of probability density pin the lattice for $|\psi(-)|^{2} \propto \sin ^{2}(\pi x / a) \quad$ (solid line), $|\psi(+)|^{2} \propto \cos ^{2}(\pi x / a)$ (dotted line) and for a traveling wave (one point broken line) [7]. charge distributions, it is in the nature of things that potential energy $\rho(+)$ is lower than that of the traveling wave, whereas the potential energy of $\rho(-)$ is higher than that of the traveling wave. If energy difference between $\rho(+)$ and $\rho(-)$ is equal to $E_{g}$, then an energy gap of width becomes $E_{g}$ Assuming that the potential energy in electron in the crystal at the point $x$ could be expressed by

$$
U(x)=U \cos (2 \pi x / a),
$$

the energy difference between two standing wave states is

$$
\begin{gathered}
E_{g}=\int_{0}^{1} \mathrm{~d} x U(x)\left\{|\psi(+)|^{2}-|\psi(-)|^{2}\right\} \\
=2 \int \mathrm{d} x U \cos (2 \pi x / a)\left(\cos ^{2} \pi x / a-\sin ^{2} \pi x / a\right)=U .
\end{gathered}
$$

It is found that the gap is equal to the Fourier component of the crystal potential, which is the off-diagonal term in the matrix $\left(\mathrm{S}_{\mathrm{ij}}\right)$ in the secular Equation (4) and can cause an insulator, a metal, a semimetal etc in the above. In DTXD, by use of $\boldsymbol{k}_{g}=\boldsymbol{k}_{\mathrm{o}}+\boldsymbol{g}$, the amplitudes of the Bloch waves in the section 2 [5] could be given by

$$
\begin{gathered}
\boldsymbol{d}(\boldsymbol{r})=\boldsymbol{d}_{o} \exp \left(-i \boldsymbol{k}_{o} \cdot \boldsymbol{r}\right)+\boldsymbol{d}_{g} \exp \left(-i \boldsymbol{k}_{g} \cdot \boldsymbol{r}\right) \\
=\left\{\boldsymbol{d}_{o}+\boldsymbol{d}_{g} \exp (-i \boldsymbol{g} \cdot \boldsymbol{r})\right\} \exp \left(-i \boldsymbol{k}_{o} \cdot \boldsymbol{r}\right) .
\end{gathered}
$$

From this, the intensity of the wave-field can be given by

$$
I=d^{2}=d_{\mathrm{o}}^{2}\left\{1+R^{2}+2 R C \cos (\boldsymbol{g} \cdot \boldsymbol{r})\right\},
$$

in which the amplitude ratio is represented by

$$
R=d_{g} / d_{o}=\alpha_{o} / C K \chi_{\bar{g}}=C K \chi_{g} / \alpha_{g}
$$

where $\alpha_{\mathrm{o}}=(K / 2)\left\{\boldsymbol{k}_{\mathrm{o}} \cdot \boldsymbol{k}_{\mathrm{o}}-k^{2}\left(1+\chi_{o}\right)\right\}$

and

$$
\alpha_{g}=(K / 2)\left\{\boldsymbol{k}_{g} \cdot \boldsymbol{k}_{g}-k^{2}\left(1+\chi_{g}\right)\right\}
$$

from off-diagonal term in the matrix $\left(\mathrm{S}_{\mathrm{ij}}\right)$. For simplicity, instead of pile up of electrons, the wave field intensity is modulated by the factor $\cos (\boldsymbol{g} \cdot \boldsymbol{r})$ in Equation (16), which has maxima at $\boldsymbol{g} \cdot \boldsymbol{r}=n$. It corresponds to an atomic plane and minima at $g \cdot r=(2 n+1) / 2$ with integral $n$. Therefore, by giving a different reading $r$ instead of $x$ in Figure 4(b), the maxima and minima of the standing wave occur at or halfway between the atomic planes. Including a role of the polarization factor $C$, it is important to stress that all of the off-diagonal terms in Equation (2) are transparent to X-rays as aforementioned and never construct the forbidden energy gaps in Figure 1 by splitting of the energy bands. The existence of the off-diagonal terms in Equation (2) could give energy dispersive $\mathrm{X}$-ray diffraction depending on the ratios from 
transmission to absorption due to the photoelectric effect. For example, in the 200 reflection in $\mathrm{NaCl}$, the structure factor $F_{g}$ is positive. Hence, $\chi_{g}$ is negative and the wave-field in branch 2 is absorbed more strongly than that in branch 1shown in Figure 5. The effect is well known as the Borrmann effect [5].

\section{Results and Discussion}

In DTXD, the asymmetric dispersion surfaces shown by solid line in Figure 1 that could be composed of hyperbolic branch and elliptic arcs have been aggressively camouflaged with the gappy dispersion surfaces devised from the crude approximation beyond the fundamental algebra and geometry in Equation (13b) mentioned mainly the Sections 3 and 4 in which they have been constructed out of the quadratics crumbled from the bi-quadratic equation based upon the crude approximated relation in Equation (12). It means that these surfaces have been lacking fundamental consistency based upon the physical necessity. Therefore, the branch 2 has not the intrinsic asymptotes, because the root is from a part of the vertex of the ellipse. Hence, the previously widespread analyses in DTXD by use of Equation (13b) should be reexamined based upon the new gapless dispersion surfaces in Figure 3(a) in Equation (7).

From Equation (14), another canonical form of the hyperbola in Equation (13b) could be represented as follows,

$$
\left(\frac{2}{K C \chi_{g}}\right)^{2} \cdot\left(k_{\mathrm{y}}^{2} \cos ^{2} \theta_{\mathrm{B}}-k_{\mathrm{x}}^{2} \sin ^{2} \theta_{\mathrm{B}}\right)=1,\left(0<\theta_{\mathrm{B}}<\pi / 2\right) .
$$

It is characteristic that the inverse of the factor in the first parentheses Equation (18) is equal to very large value as mentioned in the section 3 and the dispersive terms in the second factors consisting of two terns in parentheses are remarkably modulated by the sine squared $\theta_{\mathrm{B}}$ plus cosine squared $\theta_{\mathrm{B}}$ in Figure 6. In the region of $0<\theta_{\mathrm{B}}<\pi / 4$, the gradients of the hyperbola are steep and the intersectional angles of the asymptotes are the acute angles. And the major axis of the reference ellipse curve of $\boldsymbol{k}_{\mathrm{y}}^{2} \cos ^{2} \theta_{\mathrm{B}}+\sin ^{2} \theta_{\mathrm{B}}=1$ is parallel to the $\boldsymbol{k}_{y}$-axis. When $\theta_{\mathrm{B}}=\pi / 4$, it becomes the rectangular hyperbola and the reference curve is a circle. Moreover, in $\pi / 4<\theta_{\mathrm{B}}<\pi / 2$, those intersectional angles become the obtuse angles. That of the offered ellipse for reference is parallel to the $k_{x}$-axis.

Whereas, from Equation (9), the canonical form of the hyperbola and ellipse in Equation (8) could be represented as

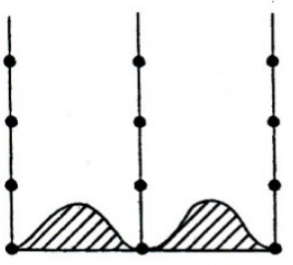

BRANCH 1

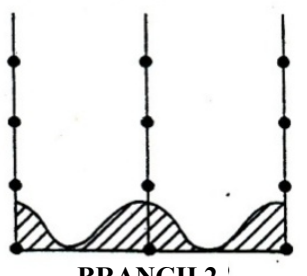

BRANCH 2
Figure 5. Intensity of the two wave-fields at the exact Bragg condition in the 200 refection. Note that branch 1 and 2 waves have a minimum intensity and a maximum one at the atomic positions, which are analogous to the electrons in Figure 4(b).

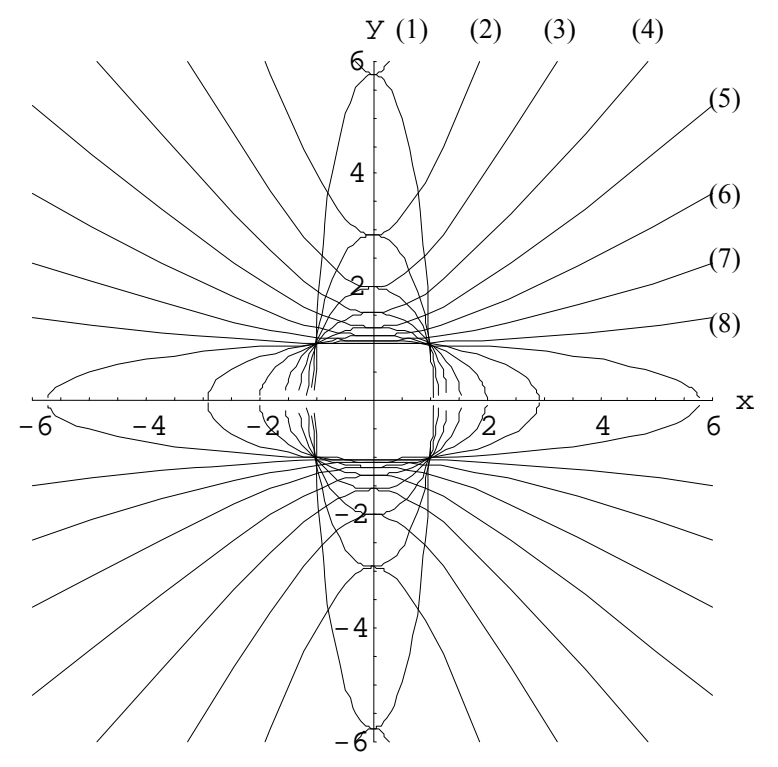

Figure 6. The main part of the dispersion surfaces of $k_{\mathbf{y}}^{2} \cos ^{2} \boldsymbol{\theta}_{\mathbf{B}}-k_{\mathbf{x}}^{2} \sin ^{2} \boldsymbol{\theta}_{\mathbf{B}}=1$ in Equation (18), together with the ellipses of $k_{\mathbf{y}}^{2} \cos ^{2} \theta_{\mathbf{B}}+k_{\mathbf{x}}^{2} \sin ^{2} \theta_{\mathbf{B}}=1$ as a reference. The Bragg angles from (1) 10 to $8(80)$ in every ten degree should be assigned to the steep slope hyperbola to the gentry slope one in order.

$$
\left(\frac{k_{y}}{K \sqrt{C \chi_{g}}}\right)^{2} \pm\left(\frac{k_{x}}{\sqrt{2} K^{2} C \chi_{g} / g}\right)^{2}=1,(0<g<2 k) .
$$

The major axis of ellipse in Equation (19) can be constantly parallel to the $k_{y}$-axis because it is constantly larger than the minor axis like $\sqrt{2} K^{2} C \chi_{g} / g<K \sqrt{C \chi_{g}}$ since $K \cong g$ in the order of magnitude in the region of $0<g<2 k$, which could corresponds to the region of the Bragg angles of $0<\theta_{B}<\pi / 2$ in Figure 2. It is considered that appearance of the scattering vector $g$ only in the parameter $a$ in Equation (9) could be appropriate. Further the gradient of the asymptotes of the hyperbola in Equation (19) can be represented as follows, 


$$
\pm(\mathrm{b} / \mathrm{a})= \pm \sqrt{\frac{2}{C \chi_{g}}} \sin \theta_{B} .
$$

If the Bragg angle $\theta_{B}$ is larger than $0.5^{\circ}$ by a rough estimation of the above expression, the intersectional angles of the asymptotes could be the acute angle and reasonable.

Conclusively, it is very important to promote that the afore-mentioned misapplication of Equitation (13b) should be reasonably set to rights and validity of the new proposed gapless dispersion surfaces in Equation (19) and should be strictly examined by thorough investigation.

\section{Acknowledgments}

The author would like cordially thanks to Prof. Dr. T. Fukamachi, Associate Prof. Dr. R. Negishi and the late M. Yoshizawa in SIT for useful stimulating discussions. He is also indebted to Emeritus Prof. Dr. H. Kobayakawa of KEK for useful suggestion and drawings of Figures 2, $\mathbf{3}$ and $\mathbf{6}$ by Mathematica.

\section{References}

[1] R. M. Stern, J. J. Perry and D. S. Boudreaux, Reviews of Morden Physics, Vol. 41, 1969, pp. 275-295. doi:10.1103/RevModPhys.41.275

[2] N. Kato, Kaisetsu and Sanran (in Japanese), "Diffraction and Scattering," Chapter 9, Toyko, 1978.

[3] A. Authier, "Dynamical Theory of X-Ray Diffraction," Chapter 4, Oxford university press, New York, 2001.

[4] Z. G. Pinsker, "Dynamical Scattering of X-Rays in Crystals," Springer-Verlag, Berlin, 1978.

[5] B. K. Tanner, "X-Ray Diffraction Topography," Pergamon Press, Oxford, 1976.

[6] S. Miyake, X sen no Kaisetsu (in Japanese), "X-Ray Diffraction," 3rd Edition, Chapter 5, Tokyo, 1988.

[7] C. Kittel, "Introduction to Solid State Physics," 6th Edition, Chapter 7, John Wiley \& Sons, Inc., New York, 1986.

[8] T. Nakajima, ECM26, Darmstadt, 2010.

[9] T. Nakajima, XTOP, Warwick, 2010.

[10] R. Kubo and T. Nagamiya, "Solid State Physics," Chapter 4, McGraw-Hill Book Co., Inc., Hong Kong. 